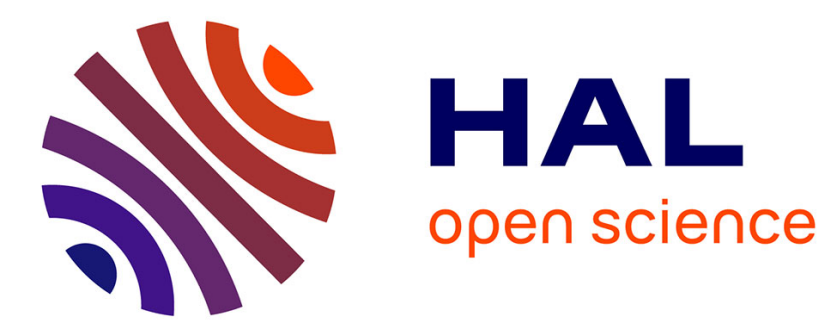

\title{
Divergence-Free Motion Estimation
}

Isabelle Herlin, Dominique Béréziat, Nicolas Mercier, Sergiy Zhuk

\section{To cite this version:}

Isabelle Herlin, Dominique Béréziat, Nicolas Mercier, Sergiy Zhuk. Divergence-Free Motion Estimation. ECCV 2012 - European Conference on Computer Vision, Oct 2012, Florence, Italy. pp.15-27, 10.1007/978-3-642-33765-9_2 . hal-00742021

\section{HAL Id: hal-00742021 \\ https://hal.inria.fr/hal-00742021}

Submitted on 15 Nov 2013

HAL is a multi-disciplinary open access archive for the deposit and dissemination of scientific research documents, whether they are published or not. The documents may come from teaching and research institutions in France or abroad, or from public or private research centers.
L'archive ouverte pluridisciplinaire HAL, est destinée au dépôt et à la diffusion de documents scientifiques de niveau recherche, publiés ou non, émanant des établissements d'enseignement et de recherche français ou étrangers, des laboratoires publics ou privés. 


\title{
Divergence-free motion estimation
}

\author{
Isabelle Herlin ${ }^{1,2}$, Dominique Béréziat ${ }^{3}$, Nicolas Mercier ${ }^{1,2}$, and Sergiy Zhuk ${ }^{4}$ \\ ${ }^{1}$ INRIA, B.P. 105, 78153 Le Chesnay, France \\ ${ }^{2}$ CEREA, joint laboratory ENPC - EDF R\&D, Université Paris-Est, 77455 Marne la \\ Vallée Cedex 2, France \\ ${ }^{3}$ Université Pierre et Marie Curie, 75005 Paris, France \\ ${ }^{4}$ IBM Research, Dublin Tech. Campus, Damastown, Dublin 15, Ireland.
}

\begin{abstract}
This paper describes an innovative approach to estimate motion from image observations of divergence-free flows. Unlike most stateof-the-art methods, which only minimize the divergence of the motion field, our approach utilizes the vorticity-velocity formalism in order to construct a motion field in the subspace of divergence free functions. A 4DVAR-like image assimilation method is used to generate an estimate of the vorticity field given image observations. Given that vorticity estimate, the motion is obtained solving the Poisson equation. Results are illustrated on synthetic image observations and compared to those obtained with state-of-the-art methods, in order to quantify the improvements brought by the presented approach. The method is then applied to ocean satellite data to demonstrate its performance on the real images.
\end{abstract}

\section{Introduction}

A fluid is called incompressible if its velocity field has zero divergence. A fluid is said incompressible if its motion is characterised by a null divergence. For instance, atmosphere and ocean are such incompressible fluids that are daily observed by a large number of satellites providing $2 \mathrm{D}$ observations of these systems. The 2D incompressible hypothesis still remains a good approximation for ocean satellite sequences if no or small vertical motion occurs (no upwelling and downwelling). This is the geostrophic assumption. Introducing the divergencefree heuristics for motion estimation methods is then a promising issue for such data sequences.

If the divergence-free assumption is assumed to be valid on an image sequence, it should be implemented through the whole computational process. However, in most of image processing methods, the velocity field $\mathbf{w}$ is estimated by solving a brightness transport equation with additional regularisation terms. In order to satisfy the divergence-free hypothesis, these terms constrain the divergence to be as small as possible, but its value is not zero. In the data assimilation framework, motion is estimated as a compromise between heuristics on the dynamics of $\mathbf{w}$ and the image observations [1]. If the motion field is divergence-free, it is then only characterised by its vorticity $\xi$, according to the Helmholtz orthogonal decomposition [2]. In this paper, we then propose to replace the heuristics on the dynamics of $\mathbf{w}$ by their equivalent on the vorticity 
$\xi$. As temporal integration of vorticity requires an additional knowledge of the velocity field, an algebraic method is described, based the projection of vorticity on a reduced basis, that converts vorticity to velocity. The divergence-free motion estimation problem is then formalised as a cost function to be minimised. Its gradient is computed from an adjoint variable [3]. The output is the vorticity field computed over the whole assimilation window, corresponding to the input image sequence. The motion field is obtained from that vorticity field solving the Poisson equation.

During the last two decades, many authors investigated the issue of fluid flow motion estimation, see for instance [4] for a survey. On one hand, transport brightness equations, based on fluid flow laws, have been proposed as alternatives to the famous brightness constancy assumption [5]. For instance, a $2 \mathrm{D}$ brightness transport equation may be derived from the 3D continuity equation in radiography fluid flow imagery [6,7]. The $2 \mathrm{D}$ continuity equation has also been proposed due to its robustness to rotational motion [8,9]. For Sea Surface Temperature (SST) oceanographic images, a 2D brightness transport equation is derived from a 3D model of ocean surface temperature [10]. On another hand, regularisation techniques, dedicated to fluid motion estimation, have been intensively studied. On 2D image sequences, a notable result is due to Suter [11], which proposed to restrain the divergence and the curl of $\mathbf{w}$ or their variations to be as small as possible. Each term having its own weight value, the user decides to constrain the divergence or/and the vorticity to be either low value or spatially regular. Suter's solution is computed with a variational technique and a B-spline decomposition. Additionally, Isambert et al. [12] proposed a Bspline multi-scale approach and a partition of unity to define control points, used to derive the solution. A multi-resolution div-curl regularisation combining Markov Random Field and Gauss-Seidel relaxation is described in [13]. The div-curl regularisation has also been used for 3D images of fluid flow [14,15], on which the incompressible assumption is verified. In [14], 3D velocity is computed from 3D Cine CT images using a $L_{2}$ regularisation under divergence-free constraint. In [15], motion is computed with a 3D div-curl regularisation function and stochastic models. To constrain motion having exact null divergence, alternatives to div-curl regularisation are proposed in the literature. Ruhnau et al., in [16], solves the optical flow equation under the constraints of Stokes equation and null divergence. Amimi, in [6], characterises the divergence-free motion as deriving from a stream function that verifies the optical flow equation.

More recently, variational data assimilation methods were applied to estimate motion using a dynamic equation on the velocity field. Ruhnau et al., in [17], define a filtering method, based on an evolution equation of vorticity. The vorticity being initialised with a null value at $T=0$, the method minimises, at each observation date, an energy function under the constraint of null divergence. This function includes three terms: optical flow equation, spatial regularity of vorticity, and coherency with the evolution equation of vorticity. The authors explain that estimations are reliable after around ten observations, which makes the method not usable for shorter sequences. In [18], velocities and temperature 
are computed from Shallow-Water and transport equations and temperature values are compared to SST image acquisitions. The velocity field is regularised with a second order div-curl norm. In [19], vorticity and divergence are both components of the state vector. The vorticity dynamics is described by a $2 \mathrm{D}$ approximation of the Navier-Stokes equations, that requires the simultaneous knowledge of velocity and vorticity. The divergence is supposed to be function of a Gaussian random variable and the authors use the heat equation to describe its dynamics. The computation of motion from vorticity and divergence is then performed in the Fourier domain using the Biot-Savart law. The comparison of the state vector with the image observations is achieved by the optical flow equation. In Papadakis et al. [20], a pure divergence-free model is defined for periodic motion field: motion is characterised by its vorticity value, which is the only component of the state vector, and the 2D Navier-Stokes equations provide the dynamic model. An error term on the dynamics is considered as a control of the optimisation problem. Images are assimilated using the optical flow equation as observation equation. The underlying assumption is that motion is constant between two consecutive acquisitions, which is however not coherent with the dynamic model.

This paper describes a divergence-free motion estimation approach, based on the Euler equations, and relying on an algebraic method to derive the motion vector from its vorticity value. The state vector $\mathbf{X}$ includes the vorticity value $\xi$ and a pseudo-image $I_{s}: \mathbf{X}=\left(\xi I_{s}\right)^{T} . I_{s}$ is supposed to have the same temporal evolution as the studied image sequence. In the paper, the heuristics of transport of grey level values by the motion field is applied. During the assimilation process, values of $I_{s}$ are compared to image observations in order to constrain the motion estimation process. The paper will discuss the impact of including the pseudo-image $I_{s}$ in the state vector on the quality of results. The assumption of Lagrangian constancy for $\mathbf{w}$ is used, from which an evolution equation of vorticity $\xi$ is derived.

Section 2 describes the divergence-free image model used for motion estimation on an image sequence. As the evolution equations involve the velocity $\mathbf{w}$, the algebraic method that computes $\mathbf{w}$ from its vorticity $\xi$ is described. Section 3 explains how the solution is obtained by minimising a cost function with a strong $4 \mathrm{D}$-Var (for a perfect model with no error on the dynamics) data assimilation method. Section 4 details the numerical aspects required for an effective implementation by interested Readers. Section 5 quantifies results on synthetic data and discusses the estimation obtained on oceanographic satellite data. Comparisons with state-of-the-art methods are provided, that justify the interest of our approach.

\section{Problem statement}

This section describes the divergence-free model, that represents motion on an image sequence. 
Let us denote $\Omega \times\left[0, t_{N}\right]$ the bounded space-time domain on which images, vorticity and motion fields are defined.

\section{$2.1 \quad$ Divergence-free model}

Vorticity characterises a rotational motion while divergence characterises sinks and sources in a flow. 2D motion $\mathbf{w}=(u v)^{T}$ is described by its vorticity, $\xi=\frac{\partial v}{\partial x}-\frac{\partial u}{\partial y}$, under the hypothesis of null divergence [2]. $\xi$ is chosen as the first component of the state vector $\mathbf{X}$ of the model. Deriving the evolution law for $\xi$ requires heuristics on the velocity $\mathbf{w}$. The Lagrangian constancy hypothesis, $\frac{d \mathbf{w}}{d t}=0$, is considered in the paper. It can be expanded as: $\frac{\partial \mathbf{w}}{\partial t}+(\mathbf{w} \cdot \nabla) \mathbf{w}=0$ :

$$
\begin{aligned}
& \frac{\partial u}{\partial t}+u \frac{\partial u}{\partial x}+v \frac{\partial u}{\partial y}=0 \\
& \frac{\partial v}{\partial t}+u \frac{\partial v}{\partial x}+v \frac{\partial v}{\partial y}=0
\end{aligned}
$$

Let us compute the $y$-derivative of Eq. (1), subtract it from the $x$-derivative of Eq. (2), and replace the quantity $\frac{\partial v}{\partial x}-\frac{\partial u}{\partial y}$ by $\xi$, we obtain:

$$
\frac{\partial \xi}{\partial t}+u \frac{\partial \xi}{\partial x}+v \frac{\partial \xi}{\partial y}+\xi\left(\frac{\partial u}{\partial x}+\frac{\partial v}{\partial y}\right)=0
$$

that is rewritten in a conservative form as:

$$
\frac{\partial \xi}{\partial t}+\nabla \cdot(\xi \mathbf{w})=0
$$

The pseudo-image $I_{s}$ is transported by motion with the same heuristics as the image sequence: this is the well known optical flow constraint equation [5], expressed as:

$$
\frac{\partial I_{s}}{\partial t}+\nabla I_{s} \cdot \mathbf{w}=0
$$

and rewritten as:

$$
\frac{\partial I_{s}}{\partial t}+\nabla \cdot\left(I_{s} \mathbf{w}\right)=0
$$

under the divergence-free hypothesis.

The model is then defined by the state vector $\mathbf{X}=\left(\xi I_{s}\right)^{T}$ and its evolution system:

$$
\begin{array}{r}
\frac{\partial \xi}{\partial t}+\nabla \cdot(\xi \mathbf{w})=0 \\
\frac{\partial I_{s}}{\partial t}+\nabla \cdot\left(I_{s} \mathbf{w}\right)=0
\end{array}
$$




\subsection{Algebraic computation of $w$}

When the state vector is integrated in time with Eqs. $(7,8)$, from an initial condition defined at date 0 , the knowledge of $\xi, I_{s}$ and $\mathbf{w}$ is required at each time step. The velocity field $\mathbf{w}$ should then be computed from the scalar field $\xi$ at each time step. A stream function $\varphi$ is first defined as the solution of the Poisson equation:

$$
-\Delta \varphi=\xi
$$

Then, $\mathbf{w}$ is derived from $\varphi$ by:

$$
\mathbf{w}=\left(\frac{\partial \varphi}{\partial y}-\frac{\partial \varphi}{\partial x}\right)^{T}
$$

In the literature (see for instance in [20]) Eq. (9) is usually solved in the Fourier domain with pseudo-spectral methods assuming periodic boundary conditions. However, this periodicity property is inadequate in our context, as there is no reason having a motion field with periodicity of the image domain's size. An algebraic solution of the Poisson equation is proposed in the following, in order to allow vorticity having Dirichlet boundary conditions with null value [21].

An eigenfunction, $\phi$, of the linear operator $-\Delta$ has to verify $-\Delta \phi=\lambda \phi$, where $\lambda$ is the corresponding eigenvalue. Explicit solutions of this eigenvalue problem are the family of bi-periodic functions $\phi_{n, m}(x, y)=\sin (\pi n x) \sin (\pi m y)$ with the associated eigenvalues $\lambda_{n, m}=\pi^{2} n^{2}+\pi^{2} m^{2}$. These functions form an orthogonal basis of a subspace of $L_{2}(\Omega)$, space of square-integrable functions defined on $\Omega$. They have null values on the domain boundary. Let $\left(a_{n, m}\right)$ be the coefficients of $\xi$ in the basis $\left(\phi_{n, m}\right): \xi(x, y)=\sum_{n, m} a_{n, m} \phi_{n, m}(x, y)$. It comes:

$$
\varphi(x, y)=\sum_{n, m} \frac{a_{n, m}}{\lambda_{n, m}} \phi_{n, m}(x, y)
$$

and eq. (9) is verified:

$$
-\Delta \varphi(x, y)=-\sum_{n, m} \frac{a_{n, m}}{\lambda_{n, m}} \Delta \phi_{n, m}(x, y)=\sum_{n, m} \frac{a_{n, m}}{\lambda_{n, m}} \lambda_{n, m} \phi_{n, m}(x, y)=\xi
$$

At each date, having knowledge of $\xi$ and $\left(\phi_{n, m}\right)$, the values of $\left(a_{n, m}\right)$ are first computed. Then $\varphi$ is derived by Eq. (11), using the $\left(\lambda_{n, m}\right)$ values.

\section{4D-Var Data Assimilation}

In order to determine $\mathbf{X}$, the $4 \mathrm{D}$-Var framework considers a system of three equations to be solved.

The first equation describes the evolution in time of the state vector $\mathbf{X}$. This is 
given by Eqs. $(7,8)$. For sake of simplicity, the system is summarised by introducing the evolution model $M$ for the state vector $\mathbf{X}$ :

$$
\frac{\partial \mathbf{X}}{\partial t}+M(\mathbf{X})=0
$$

Let us consider having some knowledge on the state vector value at initial date 0 , which is described by a background value $\mathbf{X}_{b}(x, y)$. As this initial condition is uncertain, the second equation of the system involves an error term $\epsilon_{B}$ :

$$
\mathbf{X}(x, y, 0)=\mathbf{X}_{b}(x, y)+\epsilon_{B}(x, y)
$$

The error $\epsilon_{B}(x, y)$ is supposed to be Gaussian with zero-mean and covariance function $B(x, y)$. If estimating motion from an image sequence, the only knowledge that is available is the background of the component $I_{s}$, that is chosen as the first image of the sequence: $I\left(x, y, t_{1}\right)$. The background equation, Eq. (14), reduces to:

$$
I_{s}(x, y, 0)=I\left(x, y, t_{1}\right)+\epsilon_{B_{I}}(x, y)
$$

with $B_{I}$ the part of $B$ related to $I_{s}$.

The last equation, named observation equation, links the state vector to the studied image sequence $I(x, y, t)$ : the pseudo-image $I_{s}$ has to be almost identical to the image observation $I(x, y, t)$. It is expressed as:

$$
I_{s}(x, y, t)=I(x, y, t)+\epsilon_{R}(x, y, t)
$$

Image acquisitions are noisy and their underlying dynamics could be different from the one described by Eq. (8). The observation error, $\epsilon_{R}$, is used to model these uncertainties. It is supposed Gaussian and characterised by its variance $R(x, y, t)$.

In order to discuss how Eqs. $(13,15,16)$ are solved by the data assimilation method, the state vector and its evolution equation are first approximated in time with an Euler scheme. The space variables $x$ and $y$ are further omitted for sake of simplicity. Let $d t$ be the time step, the state vector at discrete index $k$, $0 \leq k \leq N_{t}$, is denoted $\mathbf{X}(k)=\mathbf{X}(k \times d t)$. The discrete evolution equation is:

$$
\mathbf{X}(k+1)=\mathbf{X}(k)-d t M(\mathbf{X}(k))=Z_{k}(\mathbf{X}(k))
$$

with $Z_{k}(\mathbf{X}(k))=\left(\begin{array}{c}\xi(k)-d t \nabla \cdot(\xi(k) \mathbf{w}(\xi(k))) \\ \mathbf{q}(k)-d t \nabla \cdot(\mathbf{q}(k) \mathbf{w}(\xi(k)))\end{array}\right)$.

$N_{\text {obs }}$ image observations $I\left(t_{i}\right)$ are available from the image sequence, at indexes $t_{1}<\cdots<t_{i}<\cdots<t_{N_{\text {obs }}}$. Looking for $\mathbf{X}=\left(\mathbf{X}(0), \cdots, \mathbf{X}\left(N_{t}\right)\right)$ solving Eqs. $(17,15,16)$ is expressed as a constrained optimisation problem: the cost function

$$
\begin{aligned}
J(\mathbf{X})= & \frac{1}{2} \int_{\Omega} B_{I}^{-1}\left(I_{s}(0)-I\left(t_{1}\right)\right)^{2} d x d y \\
& +\frac{1}{2} \sum_{i=1}^{N_{\text {obs }}} \int_{\Omega} R^{-1}\left(t_{i}\right)\left(I_{s}\left(t_{i}\right)-I\left(t_{i}\right)\right)^{2} d x d y
\end{aligned}
$$


has to be minimised over Eq. (17). The first term of $J$ comes from Eq. (15) and the second one from Eq. (16), which is valid at observation indexes $t_{i}$.

From Eq. (17), we derive:

$$
\mathbf{X}(k)=Z_{k-1} \cdots Z_{0}[\mathbf{X}(0)]
$$

expressing that the state vector at index $k$ only depends on $\mathbf{X}(0)$. The constrained optimisation problem (18) is then rewritten as an unconstrained one: minimisation of the cost function:

$$
\begin{aligned}
J(\mathbf{X}(0))= & \frac{1}{2} \int_{\Omega} B_{I}^{-1}\left(H \mathbf{X}(0)-I\left(t_{1}\right)\right)^{2} d x d y \\
& +\frac{1}{2} \sum_{i=1}^{N_{\text {obs }}} \int_{\Omega} R^{-1}\left(t_{i}\right)\left(H Z_{t_{i}-1} \cdots Z_{0}[\mathbf{X}(0)]-I\left(t_{i}\right)\right)^{2} d x d y
\end{aligned}
$$

where $H$ stands for the projection of the state vector $X$ on its component $I_{s}$. Using calculus of variation, the gradient of $J$ is obtained from its directional derivative:

$$
\begin{aligned}
\left\langle\nabla J_{\mathbf{X}(0)}, \eta\right\rangle= & \int_{\Omega}(H \eta)^{T} B_{I}^{-1}\left(H \mathbf{X}(0)-I\left(t_{1}\right)\right) d x d y \\
& +\sum_{i=1}^{N_{\text {obs }}} \int_{\Omega}\left(H \frac{\partial Z_{t_{1}-1}}{\partial \mathbf{X}} \cdots \frac{\partial Z_{0}}{\partial \mathbf{X}} \eta\right)^{T} \times \\
& R^{-1}\left(t_{i}\right)\left(H Z_{t_{i}-1} \cdots Z_{0}[\mathbf{X}(0)]-I\left(t_{i}\right)\right) d x d y
\end{aligned}
$$

Introducing the adjoint operator, defined by $\langle A f, g\rangle=\left\langle f, A^{*} g\right\rangle$, we factorise $\eta$ in the previous equation and obtain:

$$
\begin{array}{r}
\nabla J_{\mathbf{X}(0)}=H^{T} B_{I}^{-1}\left(H \mathbf{X}(0)-I\left(t_{1}\right)\right) \\
+\sum_{i=1}^{N_{\mathrm{obs}}}\left(\frac{\partial Z_{0}}{\partial \mathbf{X}}\right)^{*} \cdots\left(\frac{\partial Z_{t_{i}-1}}{\partial \mathbf{X}}\right)^{*} H^{T} R^{-1}\left(t_{i}\right)\left(H Z_{t_{i}-1} \cdots Z_{0}[\mathbf{X}(0)]-I\left(t_{i}\right)\right)
\end{array}
$$

Let us introduce the auxiliary variable $\lambda$ defined by:

$$
\lambda(k)=\left(\frac{\partial Z_{k}}{\partial \mathbf{X}}\right)^{*} \lambda(k+1)+H^{T} R^{-1}(k)(H \mathbf{X}(k)-I(k)),
$$

$\lambda\left(N_{t}\right)=0$, and $H^{T} R^{-1}(k)(H \mathbf{X}(k)-I(k))$ being only taken into account at observation indexes $t_{i}$. It can be easily proved that the gradient reduces to:

$$
\nabla J_{\mathbf{X}(0)}=H^{T} B_{I}^{-1}\left(H \mathbf{X}(0)-I\left(t_{1}\right)\right)+\lambda(0)
$$

The cost function $J$ is minimised using an iterative steepest descent method. At each iteration, the forward time integration of $\mathbf{X}$ provides the value of $J$, then a backward integration of $\lambda$ computes $\lambda(0)$ and provides $\nabla J$. An efficient solver [22] is used to perform the steepest descent given $J$ and $\nabla J$. Full details are given in [3] about the derivation of $\nabla J$. 


\section{Numerical implementation}

The numerical scheme applied for the forward time integration of $\mathbf{X}$ is described in the following. As the evolution equations of vorticity and pseudo-image, Eqs. (7) and (8), are similar, the description is only given for the first one. A source splitting is first applied. Given a time interval $\left[t_{1}, t_{2}\right]$, we integrate successively the two equations:

$$
\begin{array}{cc}
\frac{\partial \xi^{*}}{\partial t}+\frac{\partial\left(u \xi^{*}\right)}{\partial x}=0 & t \in\left[t_{1}, t_{2}\right] \\
\frac{\partial \xi^{* *}}{\partial t}+\frac{\partial\left(v \xi^{* *}\right)}{\partial y}=0 & t \in\left[t_{1}, t_{2}\right]
\end{array}
$$

with $\xi^{*}\left(x, y, t_{1}\right)=\xi\left(x, y, t_{1}\right)$ and $\xi^{* *}\left(x, y, t_{1}\right)=\xi\left(x, y, t_{1}\right) . \xi\left(x, y, t_{2}\right)$ is then approximated as $\xi\left(x, y, t_{2}\right)=\xi^{* *}\left(x, y, t_{2}\right)+\left(\xi^{*}\left(x, y, t_{2}\right)-\xi\left(x, y, t_{1}\right)\right)$.

Let $f$ be a function defined on the space-time domain $\Omega \times\left[0, t_{N}\right]$. Let $d x$ and $d y$ be the spatial discretisation steps, supposed equal without any loss of generality: $d x=d y$. The discrete representation of $f$ is $f_{i, j}^{k}=f(i \times d x, j \times$ $d x, k \times d t)$ with $1 \leq i \leq N_{x}, 1 \leq j \leq N_{y}$ and $0 \leq k \leq N_{t}$. With these notations, Eqs. $(25,26)$ are approximated as in [23]:

$$
\begin{aligned}
& \xi_{i, j}^{*}=\xi_{i, j}^{k}-\frac{d t}{d x}\left(\left(F^{u}\right)_{i+1, j}^{k}-\left(F^{u}\right)_{i, j}^{k}\right) \\
& \xi_{i, j}^{* *}=\xi_{i, j}^{k}-\frac{d t}{d x}\left(\left(F^{v}\right)_{i, j+1}^{k}-\left(F^{v}\right)_{i, j}^{k}\right)
\end{aligned}
$$

with $F^{u}=u \xi$ and $F^{v}=v \xi$. A non-central scheme of order 3 (see [24]) is used to approximate fluxes $\left(F^{u}\right)$ and $\left(F^{v}\right)$ from the discrete representations of $\xi$ and w. $\left(F^{u}\right)_{i+1, j}^{k}$ is equal to:

$$
\begin{aligned}
& \begin{array}{r}
u_{i+1, j}^{k}\left[\xi_{i, j}^{k}+d_{0}\left(\nu_{i+1, j}^{k}\right)\left(\xi_{i+1, j}^{k}-\xi_{i, j}^{k}\right)+\right. \\
\left.d_{1}\left(\nu_{i+1, j}^{k}\right)\left(\xi_{i, j}^{k}-\xi_{i-1, j}^{k}\right)\right]
\end{array} \quad \text { if } \quad u_{i+1, j}^{k} \geq 0
\end{aligned}
$$

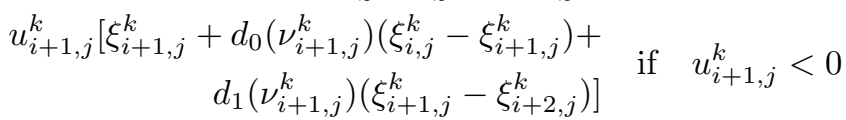

with $d_{0}(\nu)=\frac{1}{6}(2-\nu)(1-\nu), d_{1}(\nu)=\frac{1}{6}(1-\nu)^{2}$ and $\nu_{i+1, j}^{k}=\frac{d t}{d x}\left|u_{i+1, j}^{k}\right|$. The same formulation is applied for $\left(F^{u}\right)_{i, j}^{k},\left(F^{v}\right)_{i, j+1}^{k}$ and $\left(F^{v}\right)_{i, j}^{k}$.

Eqs. $(27,28)$, and those obtained from the approximation of Eq. (8), provide the discrete operator $Z_{k}$. The adjoint operator $\left(\frac{\partial Z_{k}}{\partial \mathbf{X}}\right)^{*}$ is automatically generated from the discrete operator $Z_{k}$ by an efficient automatic differentiation software (see [25]).

\section{Results}

\subsection{Synthetic experiment}

The divergence-free model is run from the initial conditions displayed in Figure 1. This provides a sequence of five synthetic observations (the first one is the initial 

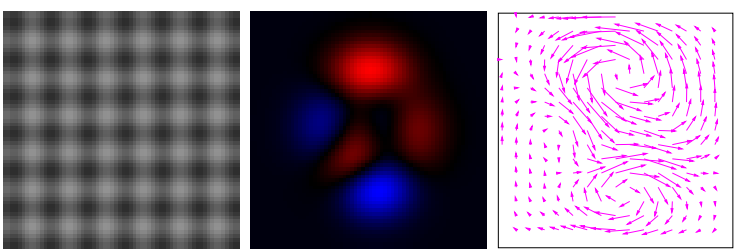

Fig. 1. Pseudo-image, vorticity and motion field at $t=0$. Positive vorticity values are coloured in red and negative one in blue.

condition and the four others are displayed on Figure 2) and the ground-truth of vorticity, motion and pseudo-image over the whole temporal window.
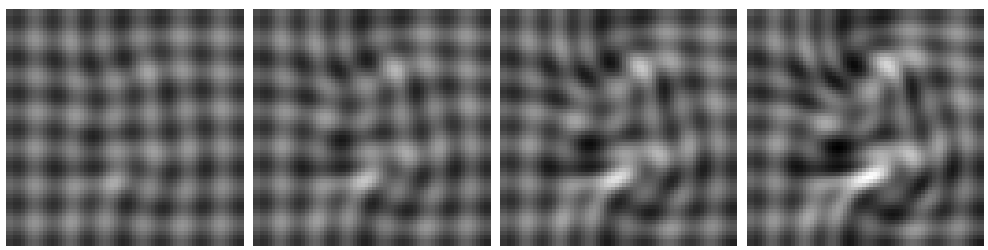

Fig. 2. Four observations of the twin experiment.

An assimilation experiment, named twin experiment, is performed with these five observations in order to retrieve the vorticity and motion fields. For that experiment, the background of vorticity is set to zero and the one of pseudoimage is the first observation. The result of the assimilation process is the state vector $\mathbf{X}(k)=\left(\xi(k) I_{s}(k)\right)^{T}$ and its associated motion vector $\mathbf{w}(k)$ over the same temporal interval than the image sequence. Statistics on the misfit between motion results and ground truth demonstrate the validity of the method: the average of the angular error and relative norm error are respectively $0.18^{\circ}$ and $0.65 \%$.

In order to compare our approach with state-of-the-art methods, a gaussian noise is added to the original observations, whose standard deviation is around one third of the image range. This provides the new observations displayed on Figure 3. In Table 1, the error between the motion result, obtained by data assimilation with these noisy images, and the ground truth is given for our approach and six state-of-the-art methods. In all cases, the optimal parameter values have been used. The first five one are image processing methods that rely on a $L_{2}$ regularisation of motion $[5,26]$ or on a second-order regularisation of the divergence $[12,13,11]$. We also compare with [20] that applies data assimilation for a divergence-free model, whose state vector reduces to vorticity, with the optical flow equation as observation equation. Results demonstrate the improvement obtained with our formalism. As the method presented in Papadakis et al. [20] is the most similar to our approach, it is important to explain why we obtain 

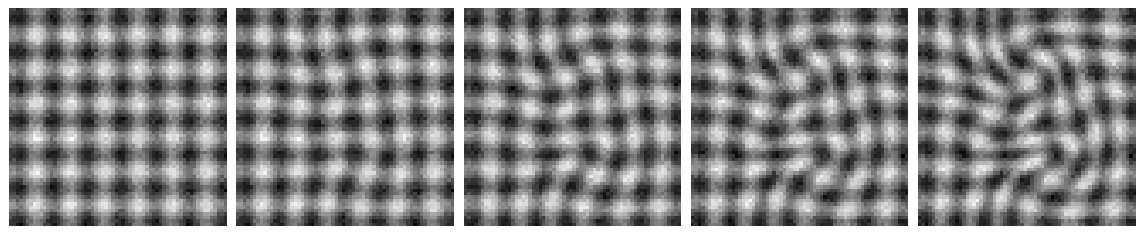

Fig. 3. Noisy observations of the twin experiment.

Table 1. Error analysis: misfit between motion results and ground truth.

\begin{tabular}{c|cc|c|c}
\hline \hline Method & \multicolumn{2}{|c|}{ Angular error (in deg. } & Relative norm error & Endpoint error \\
Mean (in \%) & Mean \\
\hline \hline Horn et al $[5]$ & 30.38 & 29.29 & 73 & 0.81 \\
Sun et al [26] & 11.31 & 12.54 & 60 & 0.6 \\
Papadakis et al [20] & 17.01 & 28.36 & 56 & 0.55 \\
Corpetti et al [13] & 7.19 & 10.78 & 26 & 0.26 \\
Isambert et al [12] & 6.71 & 14.35 & 42 & 0.37 \\
Suter [11] & 6.88 & 14.28 & 45 & 0.45 \\
Our approach & 3.32 & 10.5 & 5 & 0.04 \\
\hline \hline
\end{tabular}

better results. As said before, we assume that $N_{\text {obs }}$ image observations $I\left(t_{i}\right)$ are available at temporal indexes $t_{1}<\cdots<t_{i}<\cdots<t_{N_{\mathrm{obs}}}$. At each observation date, our observation equation is $I_{s}\left(t_{i}\right)=I\left(t_{i}\right)+\epsilon_{R}\left(t_{i}\right)$ while [20] uses:

$$
\frac{\partial I}{\partial t}\left(t_{i}\right)+\nabla I\left(t_{i}\right) \cdot \mathbf{w}\left(t_{i}\right)=\epsilon_{R}\left(t_{i}\right)
$$

The temporal gradient in Eq. (31) being computed from the image sequence, it involves at least two frames, for instance $t_{i}$ and $t_{i+1}$. Then, Eq. (31) implicitly assumes that motion is constant from $t_{i}$ to $t_{i+1}$, which is not coherent with the evolution equation (Navier-Stokes equations) of vorticity and motion used in the dynamic model. Inconsistency of equations in the data assimilation system has a negative impact on results.

\subsection{Application to oceanographic SST satellite images}

The approach has also been applied on satellite data. Observations are images acquired by NOAA/AVHRR sensors over Black Sea ${ }^{1}$, and measure the Sea Surface Temperature (SST) with a spatial resolution of about $1 \mathrm{~km}$ at nadir. In the upper layer of the Black Sea, horizontal motion is around $30 \mathrm{~cm} / \mathrm{s}$ for mesoscale eddies, while vertical motion is around $10^{-4} \mathrm{~cm} / \mathrm{s}$ and can be neglected. The 2D divergence-free assumption, or geostrophic equilibrium, is then roughly verified and the method is applicable. For the assimilation experiment, the background

\footnotetext{
${ }^{1}$ Data have been provided by E. Plotnikov and G. Korotaev from the Marine Hy-
} drophysical Institute of Sevastopol, Ukraine. 
of vorticity is set to zero and the one of pseudo-image is the first acquisition of the sequence. Two experiments are described: the first one with five observa-

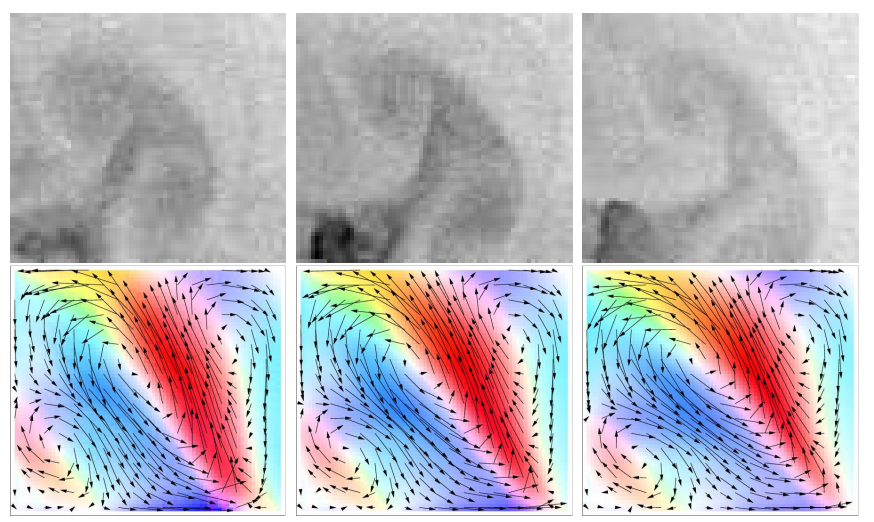

Fig. 4. Exp. 1. Observations and motion result at $t=1,3,5$.

tions (part is displayed on Figure 4) and the second one with four observations (see Figure 5). The result of motion estimation is displayed on the same figures. Visualization is made with the coloured representation tool of the Middlebury database $^{2}$, superposed with the vector representation. As explained in Section 3, the method computes the initial condition for velocity and pseudo-image that achieves the best compromise between dynamics and observations. Therefore, at acquisition dates, pseudo-images are not exactly equal to the satellite acquisitions. Their correlation measures if the structures (edges) are correctly assessed, and motion accurately estimated. Results are given in Table 2: correlation values are close to 1, proving that the motion retrieved by our method is coherent with the dynamics underlying the evolution displayed by the observations.

Table 2. Correlation between pseudo-images and observations.

\begin{tabular}{c|ccccc}
\hline \hline Date & 1 & 2 & 3 & 4 & 5 \\
\hline Experiment 1 & 0.96 & 0.94 & 0.93 & 0.94 & 0.94 \\
Experiment 2 & 0.99 & 0.93 & 0.94 & 0.97 & - \\
\hline \hline
\end{tabular}

\section{Conclusion}

The paper describes an image assimilation approach to estimate divergence-free motion on satellite acquisitions. An image model is designed: its state vector in-

\footnotetext{
$\overline{2}$ http://vision.middlebury.edu/flow/
} 

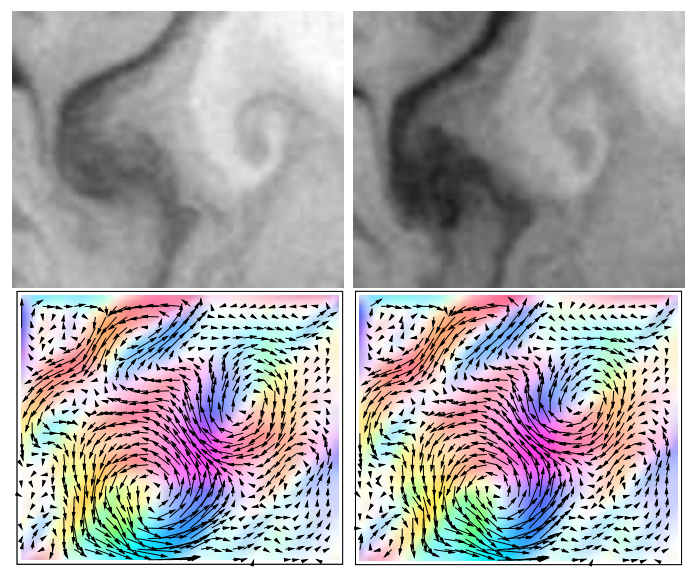

Fig. 5. Exp. 2. Observation and motion results at $t=1,3$.

cludes the vorticity and a pseudo-image, whose importance has been discussed in the results section. Motion is computed from vorticity by an algebraic method. The divergence value is then exactly null during the whole process. This allows to avoid Tikhonov regularity constraints on the divergence and the difficulty to correctly assess the constraint weights. The image assimilation technique performs a compromise between the image model and the acquired image observations in order to derive motion from an image sequence.

The method has been quantified on synthetic experiments, applied on satellite acquisitions and positively compared to well-known state-of-the-art methods.

Three main perspectives are envisaged. First, the cost of the algebraic computation of $\mathbf{w}$ from the vorticity will be decreased by limiting the set of projection fields to be taken into account for retrieving $\mathbf{w}$ from $\xi$. Second, model reduction, with a Galerkin projection on a subspace including only these projection fields, will be applied. This reduction will allow to perform data assimilation at lower cost, on long temporal assimilation windows. Last, other optimisation techniques, such as the minimax method are considered in order to also derive the estimation of uncertainty on the motion result.

\section{References}

1. Béréziat, D., Herlin, I.: Solving ill-posed image processing problems using data assimilation. Numerical Algorithms 56 (2011) 219-252 1

2. Deriaz, E., Perrier, V.: Divergence-free and curl-free wavelets in two dimensions and three dimensions: application to turbulent flows. Journal of Turbulence 7 (2006) 1-37 1, 4

3. Le Dimet, F.X., Talagrand, O.: Variational algorithms for analysis and assimilation of meteorological observations: Theoretical aspects. Tellus 38A (1986) 97-110 2, 7 
4. Heitz, D., Mémin, E., Schnörr, C.: Variational fluid flow measurements from image sequences: synopsis and perspectives. Experiments in Fluids 48 (2010) 369-393 2

5. Horn, B., Schunk, B.: Determining optical flow. Art. Int. 17 (1981) 185-203 2, 4, 9, 10

6. Amini, A.: A scalar function formulation for optical flow. In: ECCV. Volume 2. (1994) $125-1312$

7. Wildes, R., Amabile, M.: Physically based fluid flow recovery from image sequences. In: CVPR. (1997) 969-975 2

8. Del Bimbo, A., Nesi, P., Sanz, J.: Optical flow computation using extended constraints. Trans. on Image Processing 5 (1996) 720-739 2

9. Schunck, B.: The motion constraint equation for optical flow. In: ICPR. (1984) 2

10. Vigan, X., Provost, C., Bleck, R., Courtier, P.: Sea surface velocities from Sea Surface Temperature image sequences. Journal of Geophysical Research 105 (2000) 19499-19514 2

11. Suter, D.: Motion estimation and vector splines. In: CVPR. (1994) 939-942 2, 9, 10

12. Isambert, T., Herlin, I., Berroir, J.P.: Fast and stable vector spline method for fluid flow estimation. In: ICIP. (2007) 505-508 2, 9, 10

13. Corpetti, T., Mémin, E., Pérez, P.: Dense estimation of fluid flows. Pat. Anal. and Mach. Int. 24 (2002) 365-380 2, 9, 10

14. Song, S.and Leahy, R.: Computation of 3-D Velocity Fields from 3-D Cine CT Images of a Human Heart. Trans. on Medical Imaging 10 (1991) 295-306 2

15. Gupta, S.N., Prince, J.L.: On div-curl regularization for motion estimation in 3-D volumetric imaging. In: ICIP. Volume 1. (1996) 929-932 2

16. Ruhnau, P., Schnörr, C.: Optical stokes flow estimation: An imaging-based control approach. Experiments in Fluids 42 (2007) 61-78 2

17. Ruhnau, P., Stahl, A., Schnörr, C.: Variational estimation of experimental fluid flows with physics-based spatio-temporal regularization. Measurement Science and Technology 18 (2007) 755-7632

18. Huot, E., Herlin, I., Mercier, N., Plotnikov, E.: Estimating apparent motion on satellite acquisitions with a physical dynamic model. In: ICPR. (2010) 41-44 2

19. Papadakis, N., Mémin, E.: Variational assimilation of fluid motion from image sequence. SIAM Journal on Imaging Sciences 1 (2008) 343-363 3

20. Papadakis, N., Corpetti, T., Mémin, E.: Dynamically consistent optical flow estimation. In: ICCV. (2007) 1-7 3, 5, 9, 10

21. McOwen, R.: Chapter 4. In: Partial Differential Equations: Methods and Applications. Prentice Hall (2003) 5

22. Zhu, C., Byrd, R., Lu, P., Nocedal, J.: L-BFGS-B: a limited memory FORTRAN code for solving bound constrained optimization problems. Technical Report NAM11, EECS Department, Northwestern University (1994) 7

23. LeVeque, R.: Numerical Methods for Conservative Laws. 2nd edn. Lectures in Mathematics. ETH Zürich, Birkhaüser Verlag (1992) 8

24. Hundsdorfer, W., Spee, E.: An efficient horizontal advection scheme for the modeling of global transport of constituents. Monthly Weather Review 123 (1995) $3,554-3,5648$

25. Hascoët, L., Pascual, V.: Tapenade 2.1 user's guide. Technical Report 0300, INRIA (2004) 8

26. Sun, D., Roth, S., Black, M.: Secrets of optical flow estimation and their principles. In: ECCV. (2010) 2432-2439 9, 10 